\title{
Development of hydrocephalus after cervical laminoplasty for ossification of the posterior longitudinal ligament: case report
}

\author{
Yasuhisa Maezawa ${ }^{1}$, Hisatoshi Baba ${ }^{1}$, Satoru Annen ${ }^{1}$, Kenzo Uchida ${ }^{1}$, Shinichi Imura ${ }^{1}$ and Yuji Handa ${ }^{2}$ \\ ${ }^{I}$ Department of Orthopaedic Surgery; ${ }^{2}$ Department of Neurosurgery, Fukui Medical School, Shimoaizuki 23, \\ Matsuoka, Fukui 910-11, Japan
}

\begin{abstract}
We report a 69-year-old woman who developed serious hydrocephalus after cervical laminoplasty for ossification of the posterior longitudinal ligament. The patient presented with approximately 50\% spinal canal compromise pertaining to ossified lesion at C5 and C6 levels and subsequently underwent a C3-C7 open-door laminoplasty, followed by uneventful neurological recovery until 2 weeks postoperatively. Despite a favourable postoperative course, she presented with serious symptoms and signs of intracranial hypertension about 3 weeks after surgery. Computed tomography demonstrated the appearance of marked hydrocephalus, with no explainable cause. A ventriculoperitoneal shunt followed by removal of subdural fluid in the suboccipital fossa resulted in resolution of the clinical symptoms and of the hydrocephalus. It is important to be aware of the very rare occurrence of such intracranial neurological compromise after a cervical laminoplasty operation for long-standing ossification of the posterior longitudinal ligament.
\end{abstract}

Keywords: ossification; posterior longitudinal ligament; cervical spine; laminoplasty; hydrocephalus

\section{Introduction}

Osteoplastic en bloc cervical laminoplasty is an established surgical technique used for decompression of the spinal cord encroached on by ossification of the posterior longitudinal ligament (OPLL). ${ }^{1-3}$ The technique is safe and easy to employ without any injurious manipulation of the cervical cord itself, but it may be associated with an unexpected disorder of the central nervous system, requiring prompt management. In the present communication, we describe a patient who developed hydrocephalus following cervical laminoplasty for OPLL myelopathy. We also discuss the possible pathophysiological mechanisms of such neurological compromise.

\section{Case presentation}

A 69-year-old woman was admitted to our University Medical Centre suffering from a slowly progressive gait disturbance and numbness as well as clumsiness of both hands. The medical history indicated no brain disorder or haemorrhage prior to the present illness. Physical examination and blood tests on admission were unremakable except for a mild hypertension (systolic/diastolic blood pressure; 170/78 $\mathrm{mmHg}$ ), and her mental state was normal.

The patient presented with a spastic gait requiring support by crutches. Neurological examination re-

Correspondence: H Baba vealed significant hypaesthesia of both hands and the right foot, with distal predominance. The deep tendon reflexes were hyperactive in all extremities, the phantom reflexes were extensor. A gross assessment of the muscle strength was $3-4 / 5$ on upper extremities, and she showed a significant loss of the hand grasping power (right, $6.5 \mathrm{~kg}$, and left, $8.5 \mathrm{~kg}$ ). There was also a predominant right-side tetraparaesis, while bowel and bladder functions were normal.

The cervical spine films showed segmental type of OPLL spanning from C4 to C6 levels (Figure 1), with approximately $50 \%$ spinal canal compromise in the sagittal plane at C5 level. Myelography demonstrated an incomplete block at C3-C4 level, and cerebrospinal fluid (CSF) study showed slightly elevated protein level $(64 \mathrm{mg} / 100 \mathrm{ml})$ with no other abnormalities. Magnetic resonance imaging (MRI) showed an increased signal intensity on a T2-weighted sequence (Figure 2).

The patient underwent a routine $\mathrm{C} 3-\mathrm{C} 7$ en bloc open-door laminoplasty in December 1991. The surgical technique was not associated with any injurious manipulation of the cervical cord, dural laceration causing CSF leakage, or significant blood loss during surgery resulting in rapid alteration of cardiovascular parameters. The blood pressure and pulse rate were normal. The dura mater was slightly adherent to the ligamentum flavum and the inner cortices of the laminae at $\mathrm{C} 4$ and $\mathrm{C} 5$ levels, but blunt dissection was performed without technical difficulties 
and complications. The pulsation of the dura appeared satisfactory following en blocc opening of the laminae and spacer bone grafting. The spinal canal enlargement was adequate (Figure $3 \mathrm{a}$ and $\mathrm{b}$ ) and the

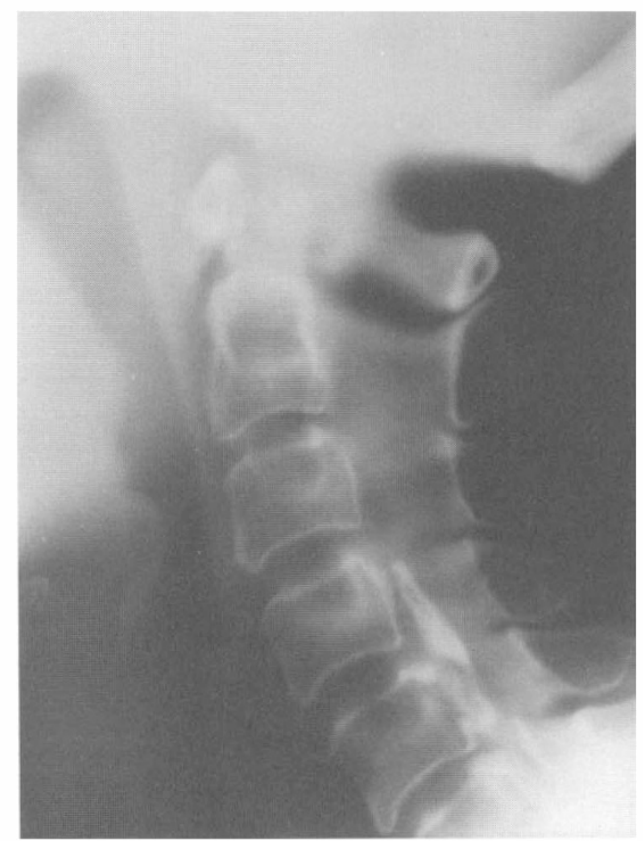

Figure 1 Lateral cervical tomograph showing segmental ossification of the posterior longitudinal ligament at C4, C5, and $\mathrm{C6}$

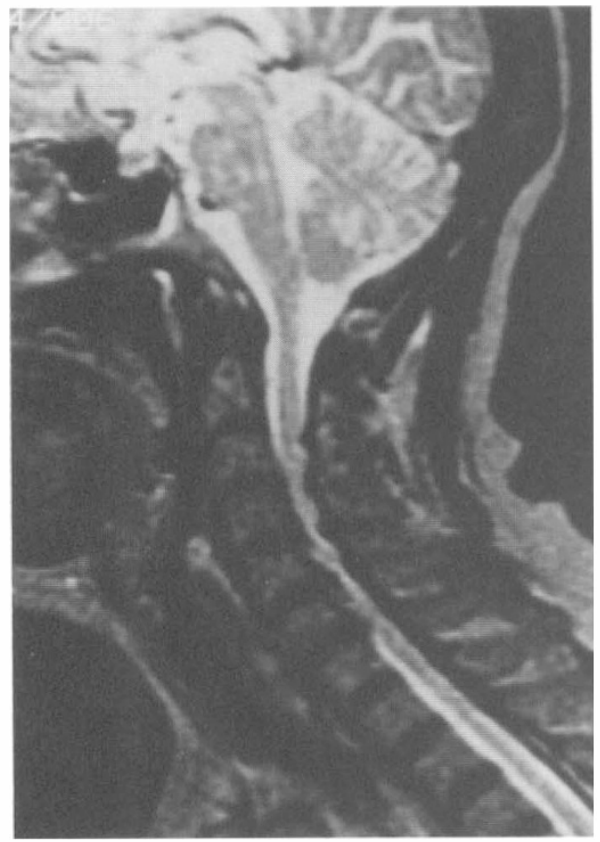

Figure 2 Magnetic resonance imaging scan showing a highintensity signal on a T2-weighted sequence (TR, $1935 \mathrm{~ms}$; TE, $100 \mathrm{~ms})$ immediate postoperative course was uneventful, with improvement of the motor paraesis and of the sensory disturbance of the extremities.

Eleven days following the operation she started to complain of headache and she was vomiting. She developed systemic hypertension $(210 / 100 \mathrm{mmHg})$ necessitating the administration of sedatives. Three weeks later her conscious level was reduced, and became semicomatose a day later. Computed tomography of the brain revealed marked enlargement of ventricular system (Figure 4), suggestive of hydrocephalus, but there was no evidence of subarachnoid or cerebral haemorrhage or of the presence of an intracranial space occupying lesion. On the following

a

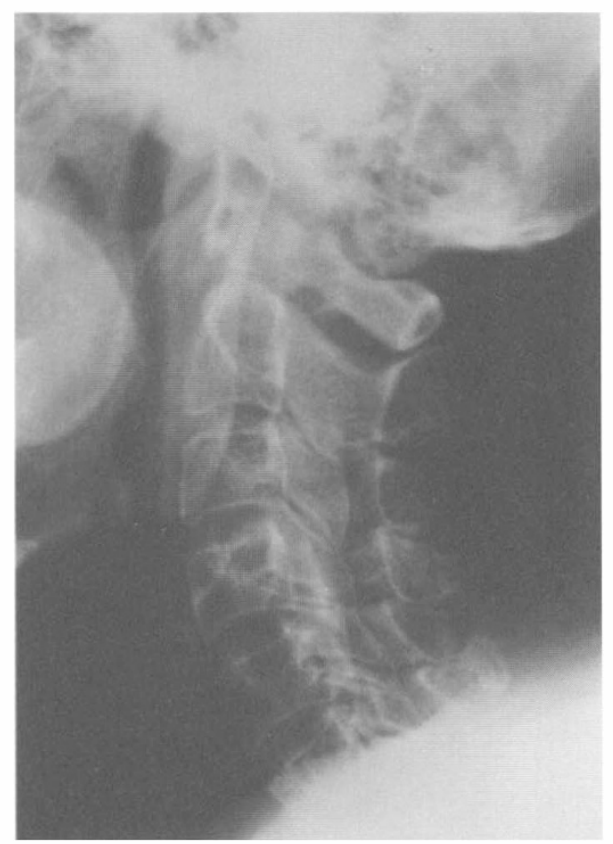

b

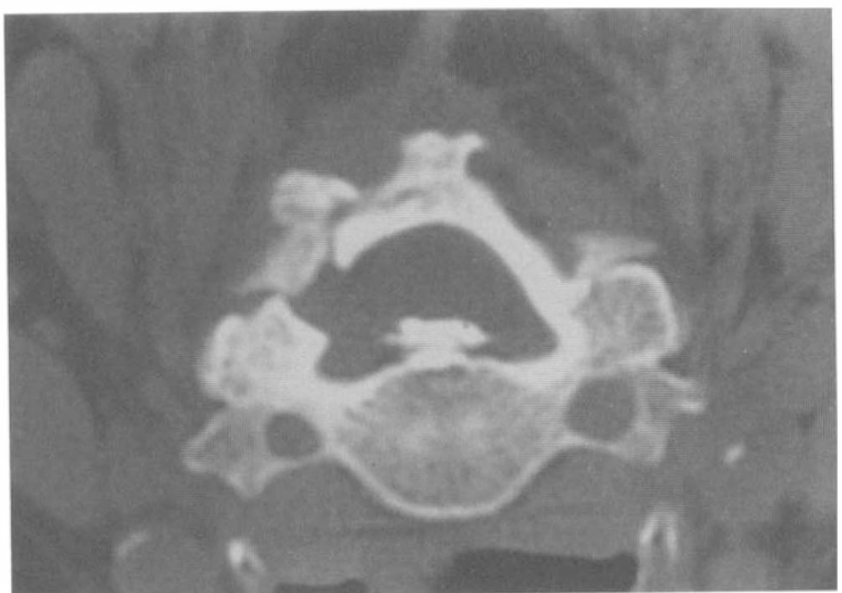

Figure 3 Postoperative lateral cervical radiograph (a) and computed tomography scan at the narrowest C5 level (b) showing enlargement of the spinal canal adequate for cord decompression 
day, she had a ventriculoperitoneal shunt operation. The CSF was clear with a low protein level of $7 \mathrm{mg} /$ $100 \mathrm{ml}$ and contained no organisms. Five days after the shunt, her mental status improved, but amnesia of recent events persisted together with disorientation. Ten days later a brain MRI scan demonstrated the presence of a subdural hydroma in the posterior cranial fossa (Figure 5); vertebral arteriography at that time did not show a vascular malformation or an aneurysm. Six days later a suboccipital craniotomy was performed to drain the subdural hydroma. The CSF was also clear at this time. The level of consciousness improved dramatically within 18 days, and computed tomography of the brain revealed reduction of the size of the ventricles and absence of fluid accumulation in the suboccipital fossa (Figure 6). Within 2 months she was ambulatory using a cane and was discharged. At the 2-year follow-up, the patient was neurologically almost normal and had a wellcontrolled blood pressure.

\section{Discussion}

Cervical OPLL myelopathy is well described and its management has been defined in recent years. ${ }^{4,5}$ In order to attain a favourable neurological recovery together with preservation of cervical spine stability, we have been treating our patients with multisegmental OPLL myelopathy (involvement of more than three vertebrae) using the osteoplastic en blocc open-door laminoplasty. $1,6,7$ We encountered only a few surgery-related complications in 127 cases treated with this procedure. These include four nerve root

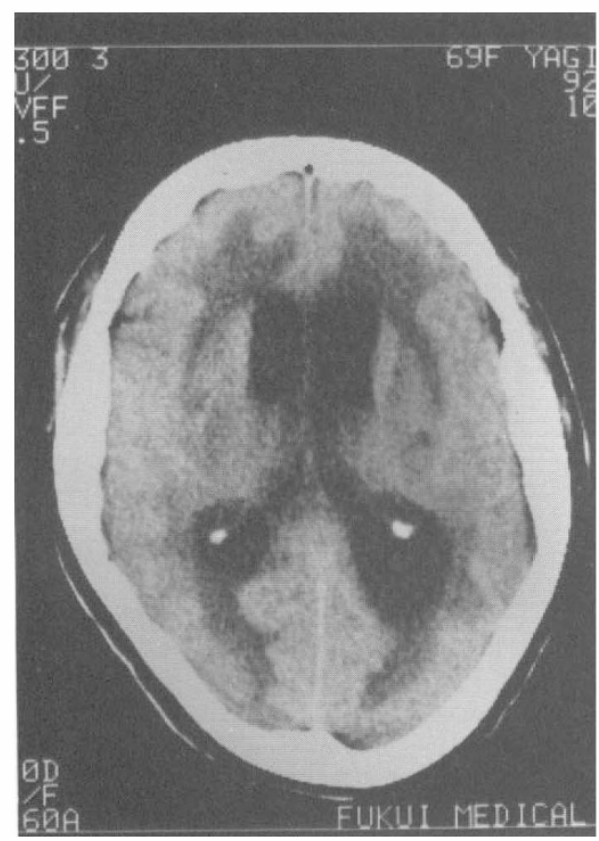

Figure 4 Computed tomography scan before ventriculoperitoneal shunt demonstrating ventricular dilatation tethering causing temporary paraesis of the arm on the laminae-opened side and three postoperative CSF leakage requiring variable duration of conservative care. The operative technique is expected to result in dorsal migration of the compromised spinal cord and maintenance of the mechanical stability of the posterior osseo-ligamentous complex necessary for neck extension. Manipulation of the spinal cord is not necessary during the operation, except for a concomitant medial foraminotomy. ${ }^{7}$ As we have previously reported, ${ }^{1,6}$ the dural sac infrequently adheres to the laminae and the ligamentum flavum at the narrowest

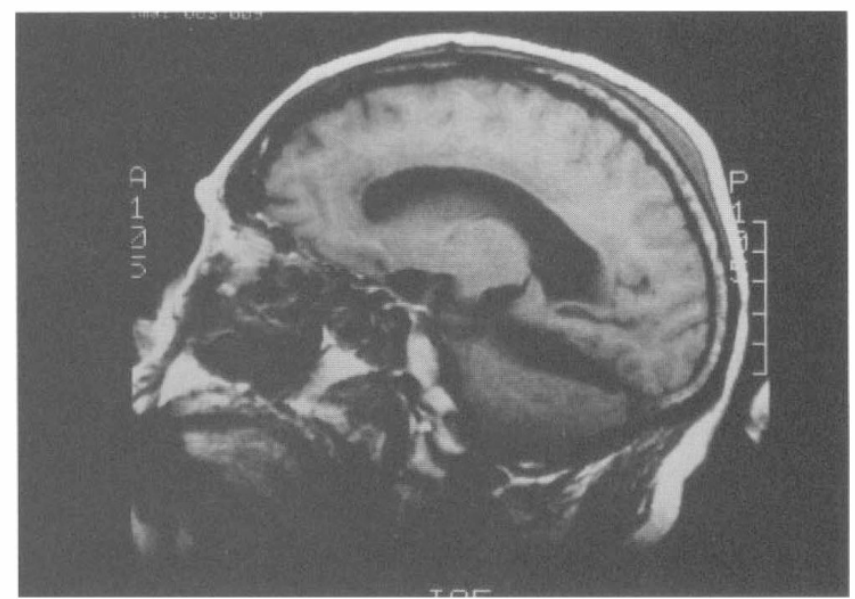

Figure 5 Magnetic resonance imaging scan showing a subdural hydroma in the posterior cranial fossa on a T2weighted sequence (TR, $1935 \mathrm{~ms}$; TE, $100 \mathrm{~ms}$ )

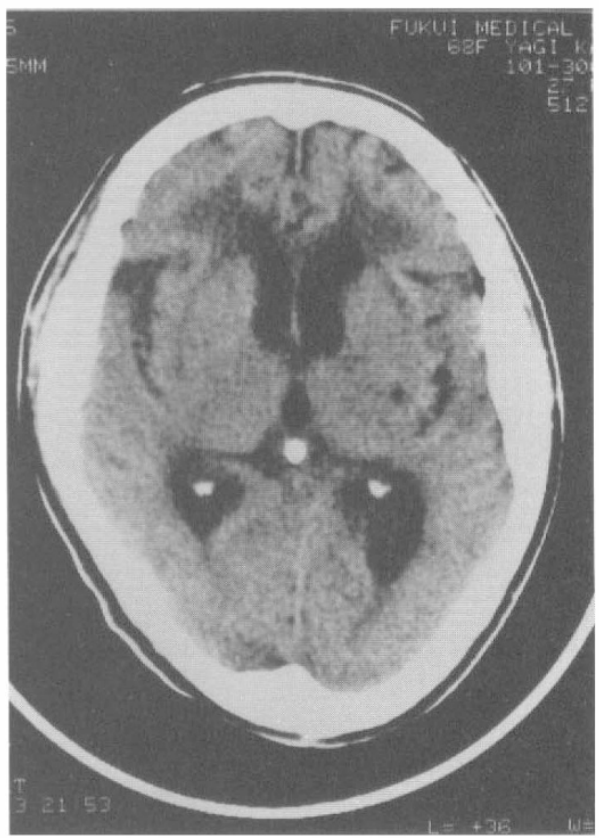

Figure 6 Computed tomography scan 35 days following surgery showing normal ventricular morphology 
spinal level but in most cases dissection is amenable to routine technique without the need of a special microsurgical technique or partial dural resection. Several groups, ${ }^{2-4}$ including ours, advocate the use of laminoplasty since it offers a safe decompression thus minimising the risk of neurological complications.

To our knowledge, the development of hydrocephalus as a complication of cervical laminoplasty has not been previously reported. Hydrocephalus may develop following a variety of spinal cord diseases, including malignancy of the spinal cord ${ }^{8-11}$ luetic granuloma ${ }^{12}$ and vascular malformation. ${ }^{13}$ Bamford et $a l^{14}$ and Bland et $a l^{15}$ reported a number of patients developing hydrocephalus after spinal cord tumour resection. These reports suggest a possible relationship between postoperative hydrocephalus and increased protein levels of CSF, spinal cord subarachnoidal haemorrhage, lengthy mechanical obstruction of CSF circulation, epidural venous congestion, and hyperviscosity of CSF, but convincing pathological mechanisms have not been provided. Harris ${ }^{16}$ also stressed the importance of increased CSF protein levels in the development of communicating hydrocephalus, and reported six illustrative cases with acoustic neurilemmomas and conus medullaris ependymoma. In his report, the CSF protein level ranged from 70 to $560 \mathrm{mg} / 100 \mathrm{ml}$. In our patient, the CSF protein level before laminoplasty was also high $(64 \mathrm{mg} / 100 \mathrm{ml})$. Such levels may potentially contribute to the development of hydrocephalus. Another possible mechanism explaining the hydrocephalus in our patient is the presence of chronic hydrocephalus that was undiagnosed on admission. Laminoplasty in turn caused a rapid accumulation of CSF. However, we did not find any clinical evidence or reported cases in the literature describing exacerbation of preoperative chronic hydrocephalus. Two additional factors may have contributed to the development of hydrocephalus in our patient. These include a prolonged and severe external mechanical compression of the spinal cord and the dura mater by the OPLL mass and/or the formation of a subdural hydroma in the suboccipital fossa. The former process may be associated with a localised arachnoiditis producing disturbance of CSF circulation. Although the preoperative MRI showed no evidence of arachnoiditis at the level of compression, prolonged impingement of the dura mater by the OPLL plaque may have contributed to a localised and mild form of arachnoiditis. Our experience indicates that most patients with a considerable magnitude of anterior cord compression by OPLL plaque show good pulsation in the dura mater after opening the laminae on one side, so that the operation technique itself is considered to improve CSF circulation when disturbed by the mass lesion. Formation of a subdural hydroma may suggest the existence of suboccipital arachnoiditis due to an inconceivable minor trauma occurring during surgery. Thus, obstruction of the CSF circulation possibly caused by suboccipital arachnoiditis may precipitate clinically evident hydrocephalus. In the present patient, subsequent improvement of the CSF circulation required a ventriculoperitoneal shunt as well as suboccipital craniotomy, resulting in neurological recovery. Thus systemic hypertension following the cervical operation and inconceivably mild suboccipital arachnoiditis may have contributed to the development of serious hydrocephalus. Unfortunately, the relationship between these factors is beyond consideration. Although the pathological mechanism is not clear, one should be aware of the very rare occurrence of such intracranial neurological compromise after cervical laminoplasty for long-standing OPLL myelopathy.

\section{References}

1 Baba $\mathrm{H}$ et al. Osteoplastic laminoplasty for cervical myeloradiculopathy secondary to ossification of the posterior longitudinal ligament. Int Orthop 1995; 19: 40-45.

2 Hirabayashi K, Satomi K. Operative procedure and results of expansive open-door laminoplasty. Spine 1988; 13: 870-876.

3 Kawai S. Cervical Laminoplasty. In: Bridwell KH, DeWald RL (eds). The Textbook of Spine Surgery. J.B. Lippincott: Philadelphia 1991, pp 805812

4 Epstein NE. The surgical management of ossification of the posterior longitudinal ligament in 51 patients. $J$ Spinal Disord 1993; 6: $432-455$.

5 McAfee PC, Regan JJ, Bohlman HH. Cervical cord compression from ossification of the posterior longitudinal ligament in nonOrientals. J Bone Joint Surg Br 1987; 69: 569-575.

6 Baba H, Furusawa N, Chen Q, Imura S. Cervical laminoplasty in patients with ossification of the posterior longitudinal ligaments. Paraplegia 1995; 33: 25-29.

7 Baba $\mathrm{H}$ et al. Laminoplasty with foraminotomy for coexisting cervical myelopathy and unilateral radiculopathy: a preliminary report. Spine 1996; 21: 196-202.

8 Gelabelt M, Bollar A, Paseio MJ, Allut AG. Hydrocephalus and intraspinal tumor in childhood. Child's Nerv Syst 1990; 6: $110-$ 112.

9 Kudo $\mathrm{H}$ et al. Intraspinal tumor associated with hydrocephalus. Neurosurgery 1987; 21: 726-731.

10 Rifkinson-Mann S, Wisoff JH, Epstein F. The association of hydrocephalus with intramedullary spinal cord tumors: a series of 25 patients. Neurosurgery 1990; 27: 749-754.

11 Zavala LM, Adler JR, Green CS, Winston KR. Hydrocephalus and intracranial tumor. Neurosurgery 1988; 22: $751-754$.

12 Fischer EG, Welch K, Shillito J. Syringomyelia following lumboureteral shunting for communicating hydrocephalus. $J$ Neurosurg 1977; 47: 96-100.

13 Mittal MM, Gupta NC, Sharma ML. Spinal epidural meningioma associated with increased intracranial pressure. Neurology 1970; 20: $818-820$.

14 Bamford CR, Labadie EL. Reversal dementia in normotensive hydrocephalus after removal of a cauda equina tumor. $J$ Neurosurg 1976; 45: 104-107.

15 Bland LI, McDonald JV. Hydrocephalus following spinal cord schwannoma resection. Arch Neurol 1992; 49: 882-885.

16 Harris P. Chronic progressive communicating hydrocephalus due to protein transudates from brain and spinal tumours. Develop Med Child Neurol 1962; 4: 270-278. 